\title{
Serum biomarkers predict adjuvant chemotherapy-associated symptom clusters in radical resected colorectal cancer patients
}

\author{
$\mathrm{Na} \mathrm{Li}^{1,2,3 \#}$, Jiajia Lu ${ }^{4 \#}$, Duanxiang Xia ${ }^{5}$, Xuetong Jiang ${ }^{3}$, Xiaomeng Wen ${ }^{2}$, Xia Qin ${ }^{2}$, Ying Chen ${ }^{1,2}$, \\ Teng Wang ${ }^{1,2}$
}

${ }^{1}$ Department of Oncology, Affiliated Hospital of Jiangnan University, Wuxi, China; ${ }^{2}$ Wuxi Medical College, Jiangnan University, Wuxi, China; ${ }^{3}$ Department of Gastrointestinal Surgery, Affiliated Suqian Hospital of Xuzhou Medical University, Suqian, China; ${ }^{4}$ Department of Internal Medicine, Wuxi No. 5 People's Hospital, Wuxi, China; ${ }^{5}$ Department of Pediatrics, Affiliated Suqian Hospital of Xuzhou Medical University, Suqian, China

Contributions: (I) Conception and design: N Li, J Lu; (II) Administrative support: T Wang; (III) Provision of study materials or patients: J Lu, D Xia; (IV) Collection and assembly of data: X Jiang; (V) Data analysis and interpretation: N Li, X Wen, X Qin; (VI) Manuscript writing: All authors; (VII) Final approval of manuscript: All authors.

\#These authors contributed equally to this work.

Correspondence to: Ying Chen. Department of Oncology, Affiliated Hospital of Jiangnan University, 1000 Hefeng Road, Wuxi 214122, China. Email: chenying126073@126.com; Teng Wang. Department of Oncology, Affiliated Hospital of Jiangnan University, 1000 Hefeng Road, Wuxi 214122, China. Email: drwangteng@163.com.

Background: Due to individual heterogeneity, patients at the same stage of colorectal cancer (CRC) who receive the same chemotherapy may experience different symptom clusters. Identifying the precise factors that predict symptom clusters is of great clinical significance for precision care and for improving the quality of life of patients. The present study investigated the relationship between serum biomarkers and adjuvant chemotherapy-related symptom clusters in radically resected CRC patients.

Methods: Serum biomarkers and clinical/pathological characteristics of the radical resected CRC patients were collected before the first cycle of adjuvant chemotherapy. A demographic questionnaire and M.D. Anderson Gastrointestinal Cancer Symptom Scale (MDASI-GI) were performed on the third day after chemotherapy and exploratory factor analysis was performed to determine the symptoms clusters. Multiple linear regression and correlation analysis were also performed to evaluate the correlation between serum biomarkers and chemotherapy-related symptom clusters.

Results: A total of 4 chemotherapy-related symptom clusters were determined in the enrolled radical resected CRC patients, including a fatigue-psychological symptom cluster, gastrointestinal symptom cluster, neurotoxic symptom cluster, and constipation-abdominal distension symptom cluster. Further analysis showed that the gastrointestinal symptom cluster was significantly associated with age, gender, weight change status, neutrophil to lymphocyte ratio (NLR), and body mass index (BMI). Additionally, the fatiguepsychological symptom cluster was found to be significantly associated with high NLR. The neurotoxic symptom cluster was found to be significantly associated with low hemoglobin level.

Conclusions: Serum biomarkers and clinical characteristics of the radical resected CRC patients could be used to predict chemotherapy-related symptoms clusters.

Keywords: Colorectal cancer (CRC); chemotherapy; serum biomarker; symptom cluster

Submitted Oct 21, 2021. Accepted for publication Jan 20, 2022.

doi: 10.21037/jgo-21-904

View this article at: https://dx.doi.org/10.21037/jgo-21-904 


\section{Introduction}

Colorectal cancer (CRC) is one of the most common malignant cancers. It is estimated that by 2030 , the global burden of CRC will increase by $60 \%$, and the number of new cases will exceed 2.2 million (1). Over recent years, the clinical outcomes of CRC patients have improved remarkably due to the improvement of surgery and systematic therapy. Although postoperative adjuvant chemotherapy has been shown to prolong disease-free survival of radical resected CRC patients, it promotes side effects in many survivors of CRC, often not a single symptom but including fatigue, restless sleep, nausea, vomiting, numbness, and other symptoms (2). These grouped symptoms have a great impact on the quality of life of CRC patients (3).

The concept of symptom clusters was first proposed by Dodd et al. (4). Symptom clusters are composed of 2 or more stable, concomitant, and interrelated symptoms, and these symptoms are independent of other symptom groups, with or without common pathogenesis (5). Due to the individual heterogeneity, patients with the same stage of CRC often receive the same chemotherapy, but experience different symptom clusters. Identifying the exact factors that can predict different symptom clusters is of great clinical significance for precision care and improving the quality of life of patients.

Previous studies have shown that systemic inflammatory response is the biological basis of symptom clusters (6), with involvement of a variety of cytokines, including interleukin (IL)-6, C-reactive protein (CRP), tumor necrosis factor $\alpha(\mathrm{TNF}-\alpha)$, and so on (7). However, these cytokines are not easily assessed by routine clinical detection. It has been shown that neutrophil-to-lymphocyte ratio (NLR), lymphocyte-to-monocyte ratio (LMR), platelet-tolymphocyte ratio (PLR), and prognostic nutritional index (PNI) $[5 \times$ lymphocyte count + serum albumin $(\mathrm{g} / \mathrm{L})]$, which can be obtained from clinical routine serum detection, could reflect systemic inflammation and predict the prognosis of CRC patients $(8,9)$. Emerging evidence $(10,11)$ has shown that the decrease of LMR and the increase of NLR and PLR before chemotherapy were associated with shorter disease-free survival (DFS) and overall survival (OS). Moreover, it has been shown that the high NLR is also associated with postoperative TNM staging. The higher the NLR value, the deeper the tumor invasion (12). The presence of multiple symptoms with chemotherapy often predicts increased mortality (13). However, most of these biomarkers are used to identify patients who can benefit from adjuvant chemotherapy and determine the prognosis of CRC patients, and their detailed relationship with chemotherapy-associated symptom clusters has not yet been clarified. The use of biomarkers to determine the symptom experience of CRC patients after adjuvant chemotherapy can be timely identified and intervened to obtain the best treatment. Therefore, in the present work, we analyzed the chemotherapy-associated symptom clusters in radical resected CRC patients and explored the potential relationship between these serum biomarkers and chemotherapy-associated symptom clusters. We present the following article in accordance with the REMARK reporting checklist (available at https://jgo.amegroups.com/ article/view/10.21037/jgo-21-904/rc).

\section{Methods}

\section{Patients}

Prospective purposive sampling was used to enroll stage I-IV CRC patients who received treatment at the Affiliated Suqian Hospital of Xuzhou Medical University from October 2019 to May 2021.The inclusion criteria were as follows: (I) patients older than 18 years of age and who signed their own informed consent form; (II) patients diagnosed with colorectal adenocarcinoma by histopathology; (III) patients who received adjuvant chemotherapy containing oxaliplatin and/or Fluoropyrimidine (FP) after radical surgery for CRC; (IV) study participants who were able to adhere to treatment as prescribed; (V) participants with no cognitive impairments and able to understand and complete their questionnaire unaided. The exclusion criteria were as follows: (I) patients who had received neoadjuvant radiotherapy or chemotherapy before surgery; (II) patients with comorbid severe hematopoietic system diseases such as aplastic anemia and coagulopathy; (III) patients with clinical evidence of active infections; (IV) patients with bowel obstruction or perforation leading to emergency surgery; (V) patients with other malignant tumors requiring concurrent treatment; (VI) patients who had used anti-infective and/or immunosuppressive drugs within 1 week.

\section{Peripheral venous blood collection and testing}

Fasting peripheral venous blood samples were collected from the radical resected CRC patients before adjuvant chemotherapy. After the blood samples were collected, they were stored at room temperature for 5-10 min, mixed well and tested within $3 \mathrm{~h}$ with reference to the routine clinical 
laboratory standards. The blood routine test was performed according to the operation procedure of SysmexXS-500i 16159 (Sysmex Corporation, Hyogo, Japan), and the blood biochemistry testing was performed according to the operation procedure of Beckman Coulter AUC680 (Beckman Coulter, Brea, CA, USA).

\section{Demographic and clinical characteristics questionnaire}

The questionnaire included the collection of general patient demographic sociological information (including gender, age, marital status, height, and weight), and clinicopathological data (including disease stage, presence or absence of stoma, chemotherapy regimen, and difference between pre-chemotherapy weight, and preoperative weight).

\section{The M.D. Anderson Symptom Inventory for Gastrointestinal Cancer (MDASI-GI)}

The severity of each the patient' symptoms was assessed using the MDASI-GI. This scale assessed the symptom experience of gastrointestinal tumor patients over the past $24 \mathrm{~h}$ and was divided into 2 major parts: the first part contained 13 core symptom items and 5 gastrointestinalspecific items, the second part was used to assess the degree of interference with life from these 18 symptoms with 0 meaning "no symptoms" or "no interference", 10 meaning "the most severe imaginable", and the higher the score, the more severe the symptoms were in general (14). The validity of this Chinese version of the scale was verified, with Cronbach's $\alpha$ of 0.84 for the symptom severity scale and 0.86 for the interference scale (15).

\section{Data collection process}

In multiple linear regression analysis, the sample size should be at least 5-10 times the number of independent variable entries. This study involved 14 independent variables, so the estimated sample size needed to be at least between 70 and 140. In summary, considering the specific clinical situation of patients as well as questionnaire recovery rate and invalid questionnaire, the final estimated sample size was 145 . The study was conducted in accordance with the Declaration of Helsinki (as revised in 2013). The study was approved by ethics board of the Affiliated Suqian Hospital of Xuzhou Medical University (No. 2019012). Informed consent was taken from all the patients. The results of routine blood examination and biochemistry were collected from the patients after surgery and before the first chemotherapy on the principle of patient consent having been provided. The on-site questionnaire was filled in on the 3rd day after chemotherapy using unified instructions and taken back on the spot.

\section{Statistical analysis}

We used R3.6.2 software (https://cran.r-project.org/bin/ macosx/) for data analysis. The Kaiser-Meyer-Olkin (KMO) measure was carried out for assessing sampling adequacy, the method of extracting symptom groups [principal component analysis (PCA) or factor analysis] was judged according to the correlation between symptoms (16). Factor extraction was based on scree plot (eigenvalues $>1$ ). When we applied factor analysis, the common factor was extracted using the principal axis iteration method (pa) with orthogonal rotations (varimax), factor loadings greater than 0.4 was considered for retention $(17,18)$, and factors with clinical explanatory significance were selected; because NLR, LMR, PLR, and PNI were calculated from neutrophil count, lymphocyte count, monocyte count, platelet, and serum albumin. In order to avoid multicollinearity, only 14 independent variables including gender, age, diagnosis, marital status, body mass index (BMI), pathological stage, presence or absence of ostomy, chemotherapy regimen, difference between weight before chemotherapy and weight before surgery, hemoglobin, NLR, LMR, PLR, and PNI were included. Multivariate linear regression equation was used to evaluate the correlation between these independent variables and the identified symptom clusters. Stepwise regression was used to obtain the model with the smallest Akaike information criterion (AIC). The best model that passed the comprehensive test of linear model assumptions was retained, and the relevant independent variables were obtained from the best model. Models that did not pass the comprehensive test of the linear model assumptions were used to analyze the correlation between outcome variables and independent variables using Pearson linear correlation coefficient. The $\mathrm{P}$ values $<0.05$ or $\mathrm{P}<0.01$ were used to indicate a significant difference.

\section{Results}

\section{Demographic and clinical characteristics with blood parameters}

A total of 145 questionnaires were distributed. There were a total of 6 patients with missing clinical information, 
Table 1 Demographic and clinical characteristics of 135 patients with CRC

\begin{tabular}{|c|c|}
\hline Characteristics & All patients $(n=135)$ \\
\hline \multicolumn{2}{|l|}{ Age (years) } \\
\hline Mean (SD) & $59.3(12.0)$ \\
\hline Median (min, max) & $59.0(29.0,84.0)$ \\
\hline \multicolumn{2}{|l|}{ Gender, n (\%) } \\
\hline Female & $47(34.8)$ \\
\hline Male & $88(65.2)$ \\
\hline \multicolumn{2}{|l|}{ Tumor location, n (\%) } \\
\hline Colon cancer & $66(48.9)$ \\
\hline Rectal cancer & $69(51.1)$ \\
\hline \multicolumn{2}{|l|}{ Married, n (\%) } \\
\hline Yes & $123(91.1)$ \\
\hline No & $12(8.9)$ \\
\hline \multicolumn{2}{|l|}{ BMI $\left(\mathrm{kg} / \mathrm{m}^{2}\right)$} \\
\hline Mean (SD) & $22.4(3.03)$ \\
\hline Median (min, max) & $22.5(15.6,31.1)$ \\
\hline \multicolumn{2}{|l|}{ Stage, n (\%) } \\
\hline I & $8(5.9)$ \\
\hline II & $51(37.8)$ \\
\hline III & $64(47.4)$ \\
\hline IV & $12(8.9)$ \\
\hline \multicolumn{2}{|l|}{ Stoma, n (\%) } \\
\hline Yes & $32(23.7)$ \\
\hline No & $103(76.3)$ \\
\hline \multicolumn{2}{|l|}{ Chemotherapy regimen, n (\%) } \\
\hline Oxaliplatin + capecitabine & $110(81.5)$ \\
\hline Capecitabine & $3(2.2)$ \\
\hline $\begin{array}{l}\text { Oxaliplatin + fluorouracil + leucovorin } \\
\text { calcium }\end{array}$ & $22(16.3)$ \\
\hline \multicolumn{2}{|l|}{ Body weight change $(\mathrm{kg})$} \\
\hline Mean (SD) & $-4.50(2.97)$ \\
\hline Median (min, max) & $-5.00(-11.0,3.00)$ \\
\hline
\end{tabular}

4 patients missed complete questionnaires, and 135 valid questionnaires were finally returned. From these, NLR, PLR, LMR, and PNI were calculated. Table 1 summarizes the demographic and clinical characteristics of the sample: $81.5 \%$ of the patients received oxaliplatin + capecitabine chemotherapy regimen, $16.3 \%$ received oxaliplatin + fluorouracil + leucovorin calcium, and $2.2 \%$ received oral capecitabine. The maximum weight loss from surgery to the first chemotherapy was $11 \mathrm{~kg}$, the biggest weight gain was $3 \mathrm{~kg}$, patients with stoma accounted for $23.7 \%$, and patients without stoma accounted for $76.3 \%$. Table 2 summarizes the results of the blood parameters.

\section{Prevalence and severity of symptoms}

Table 3 shows that drowsiness (94.1\%), fatigue (93.3\%), lack of appetite $(93.3 \%)$, and nausea $(91.9 \%)$ were the top 4 prevalent symptoms. The more severe symptoms included fatigue, lack of appetite, drowsiness, nausea, and disturbed sleep, with mean scores of $4.86(\mathrm{SD}=1.95), 4.37(\mathrm{SD}=2.07)$, $4.35(\mathrm{SD}=1.91), 4.24(\mathrm{SD}=1.99)$, and $4.03(\mathrm{SD}=2.11)$, respectively. Since this study did not examine the impact of symptom interference on quality of life, this symptom interference scale was not statistically analyzed.

\section{Symptom clusters}

There was a high correlation between certain symptoms in the sample (Figure 1), so factor analysis was used to determine the symptom cluster of patients with CRC undergoing postoperative chemotherapy. The KMO test was 0.774 , and the sample size was fair. The results of Bartley's spherical test were $\chi^{2}=1440.456, \mathrm{df}=153$, and $\mathrm{P}<0.000$, which met the requirements of factor analysis. There were 5 factors with eigenvalues $>1$ extracted (Figure 2), and the factors with factor loadings $\geq 0.4$ were retained to yield 4 symptom clusters (Table 4). The fatigue-psychological symptom cluster contained 5 symptoms: fatigue, disturbed sleep, drowsiness, upset, and sadness; the gastrointestinal symptom cluster contained 4 symptoms: nausea, lack of appetite, vomiting, and change in taste; the neurotoxic symptom cluster contained 3 symptoms: amnesia, numbness, and dry mouth; and the constipation-abdominal distension symptom cluster was constipation and feeling bloated. These 4 symptom clusters explained a total of $57 \%$ of the variance in this sample, and Figures 3,4 are visualizations of the results of the symptom clusters in this sample.

\section{Influencing factors of the symptom cluster}

All variables were included, and 4 symptom clusters 
Table 2 Blood parameters of 135 patients with CRC

\begin{tabular}{|c|c|}
\hline Blood parameters & All patients $(n=135)$ \\
\hline \multicolumn{2}{|l|}{$\mathrm{NE}\left(\times 10^{9} / \mathrm{L}\right)$} \\
\hline Mean (SD) & $3.23(1.34)$ \\
\hline Median (min, max) & $2.98(0.880,9.19)$ \\
\hline \multicolumn{2}{|l|}{ Lym (×10/L) } \\
\hline Mean (SD) & $1.55(0.506)$ \\
\hline Median (min, max) & $1.47(0.620,3.41)$ \\
\hline \multicolumn{2}{|l|}{ Mono $\left(\times 10^{9} / \mathrm{L}\right)$} \\
\hline Mean (SD) & $0.404(0.157)$ \\
\hline Median (min, max) & $0.380(0.120,0.960)$ \\
\hline \multicolumn{2}{|l|}{ PLT $\left(\times 10^{9} / \mathrm{L}\right)$} \\
\hline Mean (SD) & $228(71.1)$ \\
\hline Median (min, max) & $221(101,477)$ \\
\hline \multicolumn{2}{|l|}{$\mathrm{Hgb}(\mathrm{g} / \mathrm{L})$} \\
\hline Mean (SD) & $128(19.1)$ \\
\hline Median (min, max) & $130(66.0,169)$ \\
\hline \multicolumn{2}{|l|}{ Alb (g/L) } \\
\hline Mean (SD) & $43.8(3.48)$ \\
\hline Median (min, max) & $44.1(30.7,52.8)$ \\
\hline \multicolumn{2}{|l|}{ NLR } \\
\hline Mean (SD) & $2.22(1.04)$ \\
\hline Median (min, max) & $1.98(0.690,6.87)$ \\
\hline \multicolumn{2}{|l|}{ LMR } \\
\hline Mean (SD) & $4.23(1.60)$ \\
\hline Median (min, max) & $4.02(1.12,9.45)$ \\
\hline \multicolumn{2}{|l|}{ PLR } \\
\hline Mean (SD) & $159(70.4)$ \\
\hline Median (min, max) & $145(49.5,650)$ \\
\hline \multicolumn{2}{|l|}{ PNI } \\
\hline Mean (SD) & $51.6(4.31)$ \\
\hline Median (min, max) & $51.7(40.3,63.7)$ \\
\hline
\end{tabular}

CRC, colorectal cancer; NE, neutrophil; Hgb, hemoglobin; PLT, platelet; Alb, albumin; NLR, neutrophil-to-lymphocyte ratio; LMR, lymphocyte-to-monocyte ratio; PLR, platelet-tolymphocyte ratio; PNI, prognostic nutritional index.
Table 3 Prevalence and severity of symptoms ( $\mathrm{N}=135)$

\begin{tabular}{|c|c|c|c|}
\hline \multirow{2}{*}{ Symptoms } & \multirow{2}{*}{ Occurrence (\%)- } & \multicolumn{2}{|c|}{ Severity scores } \\
\hline & & Mean (SD) & Median (min, max) \\
\hline Pain & 21.5 & $0.33(0.80)$ & $0(0,6.00)$ \\
\hline Fatigue & 93.3 & $4.86(1.95)$ & $5.00(0,9.00)$ \\
\hline Disturbed sleep & 88.1 & $4.03(2.11)$ & $5.00(0,8.00)$ \\
\hline Drowsiness & 94.1 & $4.35(1.91)$ & $5.00(0,8.00)$ \\
\hline Upset & 88.9 & $3.32(1.87)$ & $3.00(0,8.00)$ \\
\hline Sadness & 83.0 & $2.77(1.82)$ & $3.00(0,7.00)$ \\
\hline Change in taste & 85.2 & $3.57(1.96)$ & $4.00(0,7.00)$ \\
\hline Nausea & 91.9 & $4.24(1.99)$ & $4.00(0,9.00)$ \\
\hline Lack of appetite & 93.3 & $4.37(2.07)$ & $5.00(0,8.00)$ \\
\hline Vomiting & 65.2 & $1.45(1.42)$ & $1.00(0,6.00)$ \\
\hline Amnesia & 40.7 & $0.64(0.87)$ & $0(0,3.00)$ \\
\hline Numbness & 49.6 & $0.86(1.05)$ & $0(0,4.00)$ \\
\hline Dry mouth & 49.6 & $0.87(1.01)$ & $0(0,4.00)$ \\
\hline $\begin{array}{l}\text { Shortness of } \\
\text { breath }\end{array}$ & 8.1 & $0.10(0.34)$ & $0(0,2.00)$ \\
\hline Constipation & 25.9 & $0.35(0.68)$ & $0(0,4.00)$ \\
\hline Diarrhea & 17.8 & $0.30(0.70)$ & $0(0,3.00)$ \\
\hline Swallowing & 2.2 & $0.02(0.15)$ & $0(0,1.00)$ \\
\hline Feeling bloated & 42.2 & $0.62(0.88)$ & $0(0,4.00)$ \\
\hline
\end{tabular}

were regressed, respectively. A total of 4 models were preliminarily obtained (Table 5). The model with the smallest AIC was selected by stepwise regression method, and the results are shown in Table 6. In the comprehensive test of model assumptions, only the gastrointestinal symptom cluster model met all assumptions of multiple linear regression, and the regression results only had explanatory significance for this symptom cluster model. Since the study focused on exploring the effects of nutritional and inflammatory indicators in the blood on the symptom cluster, only the fatigue-psychological symptom cluster, neurotoxicity symptom cluster, and constipationabdominal distension symptom cluster were used to analyze correlations with Hgb, NLR, LMR, PLR, and PNI (Pearson correlation), and the results are shown in Table 7. 


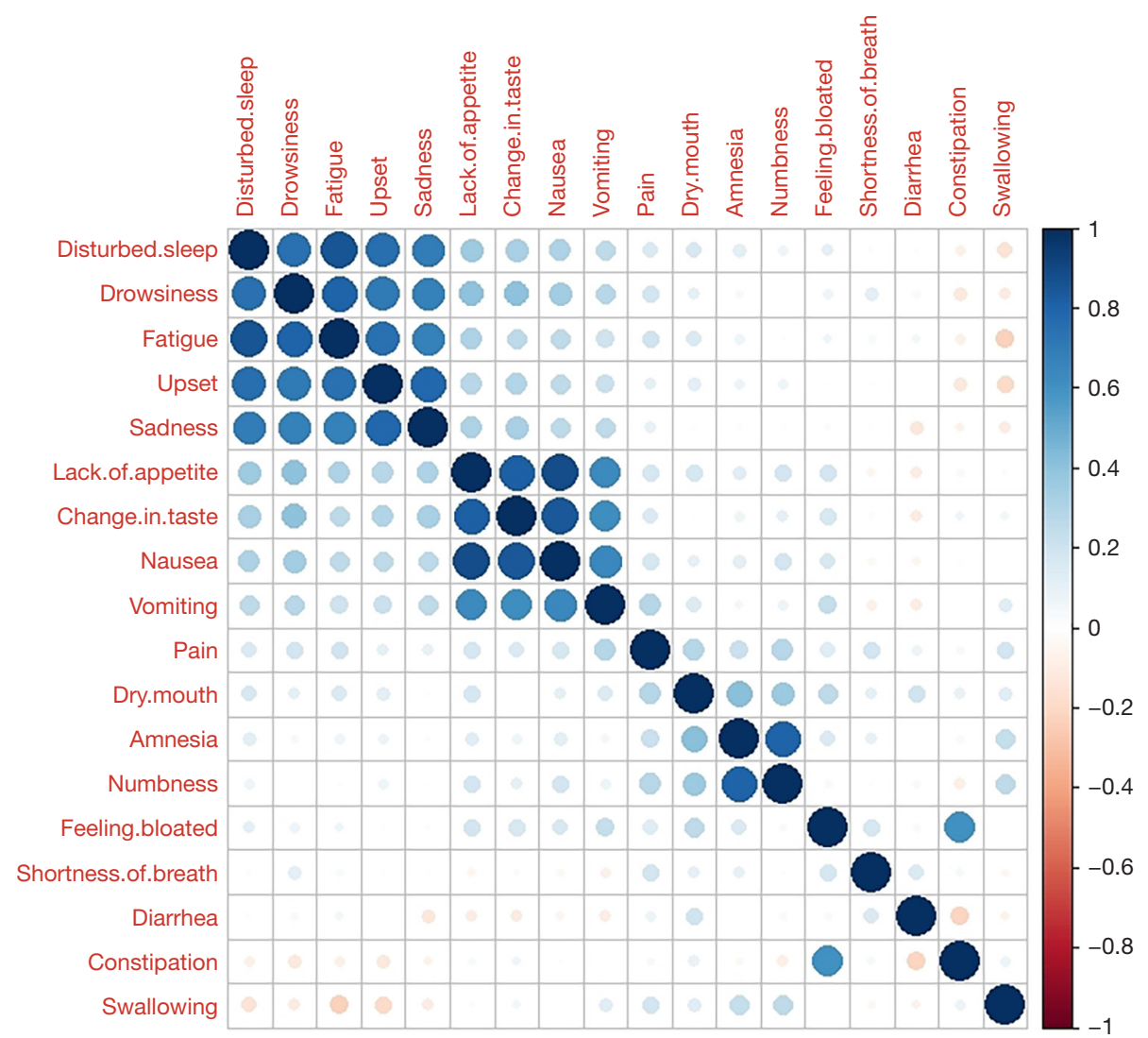

Figure 1 Correlation between symptoms.

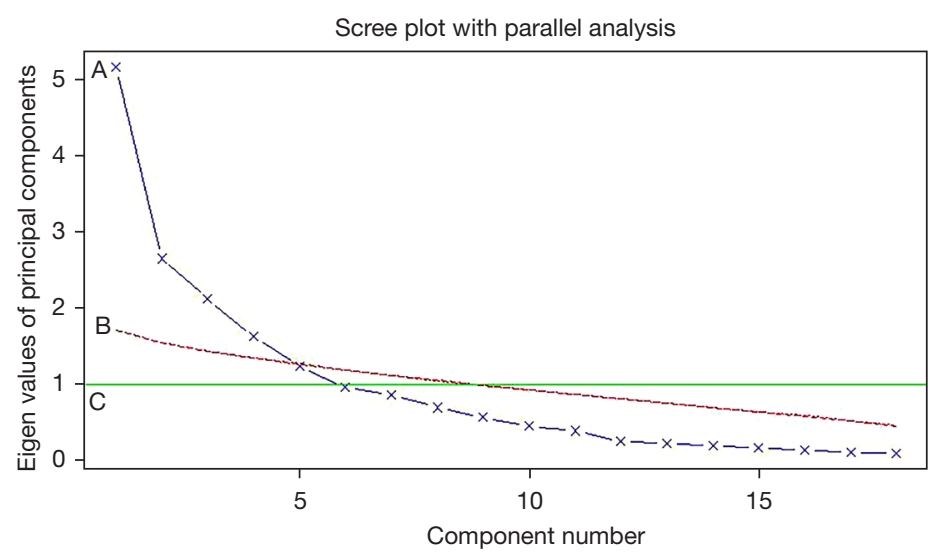

Figure 2 Lithotripsy. Line A shows the lithotripsy test based on the observed eigenvalues; line B indicates the mean of the eigenvalues derived from 100 random data matrices; and line $\mathrm{C}$ indicates the criterion of eigenvalues greater than 1.

The results of multivariate linear regression showed (Table 6) that age, gender, weight change status, NLR, and BMI were statistically significant $(\mathrm{P}<0.05)$ and were the influencing factors of gastrointestinal symptom cluster severity. The finally established regression equation was: gastrointestinal symptom cluster severity $=3.54-0.06$ * weight change status +0.16 * NLR -0.07 * $\mathrm{BMI}-0.89$ * gender $-0.03 *$ age $($ Males $=1$; Females $=0)$, and this model 
Table 4 Symptom clusters $(\mathrm{N}=135)$

\begin{tabular}{|c|c|c|c|c|}
\hline Symptoms & PA1 & PA2 & PA3 & PA4 \\
\hline Pain & 0.12 & 0.18 & 0.29 & 0.09 \\
\hline Fatigue & 0.89 & 0.13 & -0.01 & 0.01 \\
\hline Nausea & 0.15 & 0.93 & 0.09 & -0.01 \\
\hline Disturbed sleep & 0.87 & 0.19 & 0.05 & 0.03 \\
\hline Upset & 0.86 & 0.13 & 0.03 & -0.06 \\
\hline Shortness of breath & 0.03 & -0.05 & 0.04 & 0.11 \\
\hline Amnesia & 0.07 & 0.01 & 0.87 & 0.02 \\
\hline Lack of appetite & 0.21 & 0.88 & 0.12 & 0.05 \\
\hline Drowsiness & 0.80 & 0.27 & -0.03 & -0.02 \\
\hline Dry mouth & 0.10 & 0.07 & 0.43 & 0.16 \\
\hline Sadness & 0.81 & 0.16 & -0.01 & -0.01 \\
\hline Vomiting & 0.14 & 0.68 & 0.08 & 0.09 \\
\hline Numbness & 0.01 & 0.09 & 0.90 & -0.10 \\
\hline Constipation & -0.09 & 0.00 & -0.01 & 0.86 \\
\hline Diarrhea & -0.02 & -0.06 & -0.01 & -0.15 \\
\hline Swallowing & -0.19 & 0.07 & 0.31 & 0.04 \\
\hline Change in taste & 0.21 & 0.86 & 0.04 & 0.06 \\
\hline Feeling bloated & 0.01 & 0.18 & 0.09 & 0.74 \\
\hline SS loadings & 3.80 & 3.10 & 1.98 & 1.39 \\
\hline Proportion var & 0.21 & 0.17 & 0.11 & 0.08 \\
\hline Cumulative var & 0.21 & 0.37 & 0.49 & 0.57 \\
\hline
\end{tabular}

PA1 refers to the factor 1 extracted using the principal axis iteration method (pa), representing the fatigue-psychological symptom cluster; PA2 refers to the factor 2, representing the gastrointestinal symptom cluster; PA3 refers to the factor 3 , representing the neurotoxic symptom cluster; PA4 refers to the factor 4 , representing the constipation-bloating symptom cluster. Bold fonts indicate factor loading for symptom was $>0.40$.

could explain $41.3 \%$ of gastrointestinal symptom cluster severity (adjusted $\mathrm{R}^{2}=41.3 \%$ ).

Table 7 shows that there was a significant positive correlation between the fatigue-psychological symptom cluster and NLR $(r=0.63, \mathrm{P}<0.01)$, and a significant negative correlation between the neurotoxic symptom cluster and $\operatorname{Hgb}(\mathrm{r}=-0.72, \mathrm{P}<0.05)$.

\section{Discussion}

Postoperative adjuvant chemotherapy in colorectal cancer

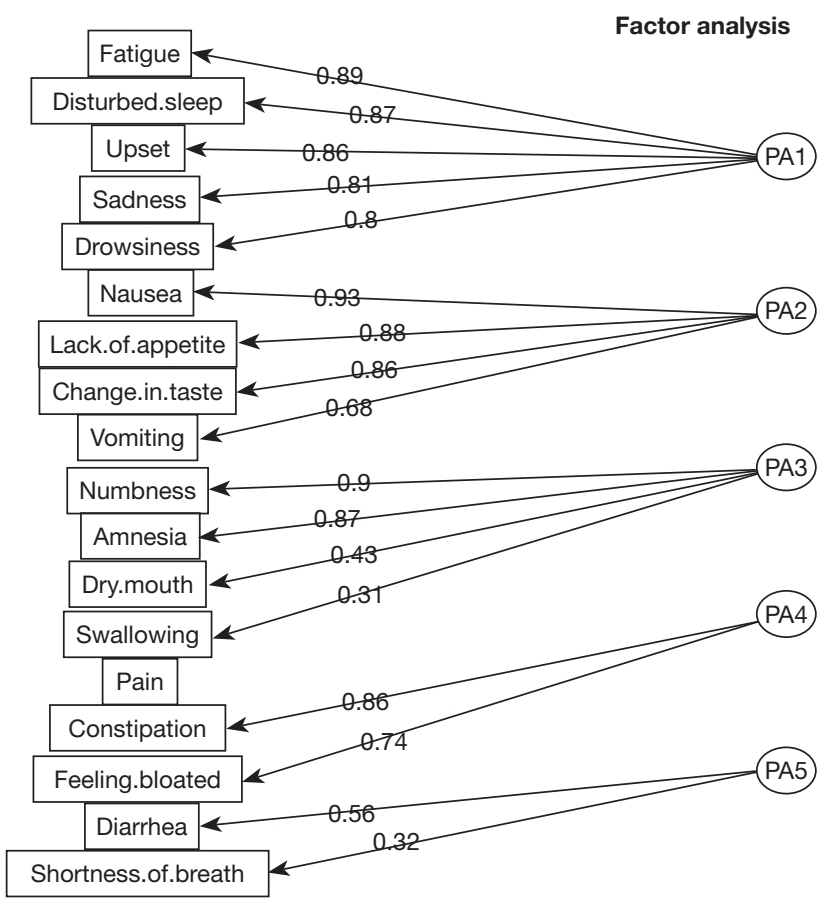

Figure 3 Visualization of symptom cluster.

patients aims to eradicate micrometastatic disease and improve survival, and it is generally accepted that 3 or 6 months of oxaliplatin-based adjuvant chemotherapy (19). DFS and OS are considered as endpoint measures to judge the efficacy of adjuvant chemotherapy (20), adjuvant chemotherapy has greatly improved the DFS and OS of CRC patients. With the development of precision medicine, clinicians need effective biomarkers to determine optimal treatment strategies for managing patients who benefit from adjuvant chemotherapy (21). Therefore, biomarkers such as mismatch repair status, immunoscore and circulating tumor DNA were used to predict specific prognosis and response to adjuvant chemotherapy in CRC patients (22). However, biomarkers to predict symptoms after adjuvant chemotherapy are lacking. Sheu et al. (23) used The MD Anderson Symptom Inventory-Head and Neck Module (MDASI-HN) baseline score as the primary predictor to predict the severity of symptoms during radiotherapy in patients with head and neck cancer, and generated nomograms.

Relevant studies $(16,17)$ have found symptom clusters in CRC patients, while the potential predictor of symptom clusters remains largely unknown. In this study, we identified 4 symptom clusters in radical resected CRC patients who had just undergone their first cycle of adjuvant 


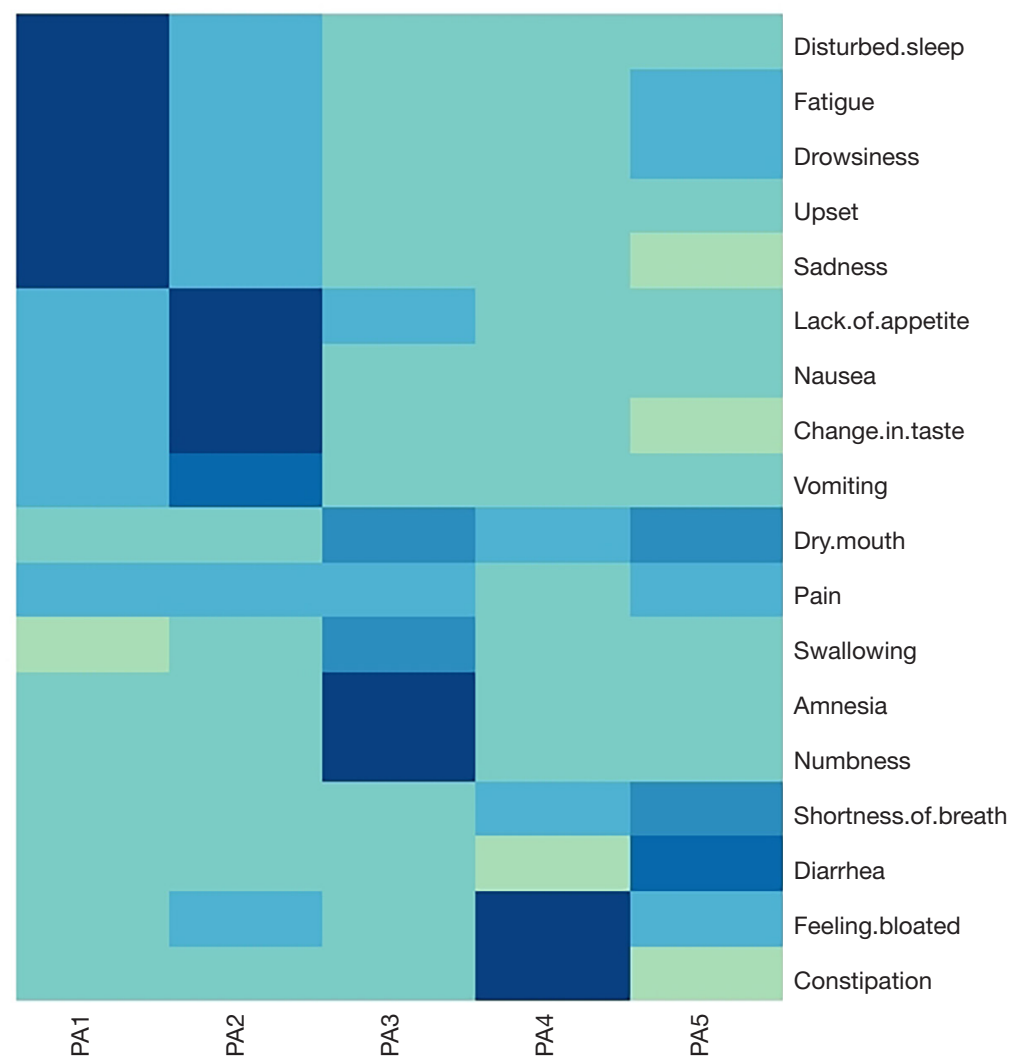

Figure 4 Visualization of symptom cluster.

chemotherapy. Inconsistent with the findings in a previous study (2), we observed different symptoms in these same symptom clusters. The possible reason might be different cycles of chemotherapy or participant characteristics.

Previous studies have revealed a close correlation between fatigue and psychological symptoms in symptom clusters study in cancer patients. An evaluation of CRC patients (24) suggested to evaluate the potential of anxiety and depression in the treatment of cancer-related fatigue in CRC patients, regardless of age. In the study of fatigue-related symptom clusters, a variety of biological mechanisms have been proposed, including cytokine imbalance, hypothalamic pituitary adrenal (HPA) axis imbalance, 5-hydroxytryptophan (5-HT) neurotransmitter imbalance, adenosine triphosphate, and muscle metabolic changes $(18,25)$. Among these, inflammation related cytokines have received much attention. It was shown that cancer and caner-related therapy could influence systematic inflammation, and then send signals to the central nervous system, resulting in fatigue and other symptoms (26). It was reported that the fatigue-related symptom cluster was associated with a variety inflammation-related cytokines, including IL-1 $\beta$, TNF- $\alpha$, IL-6, and CRP. However, these cytokines are not easily obtained through routine clinical detection. In this study, the serum biomarkers obtained from clinical routine detection which have been shown to be associated the systematic inflammation, were used to evaluate chemotherapy-related symptom clusters. We found that chemotherapy-related symptom clusters were associated with several serum biomarkers, including NLR, LMR, PLR, and so on.

As the incidence of diarrhea in this study was low, it was not included in the gastrointestinal symptom cluster (16). It might have been because of the high application of Miles' resection in the patients enrolled, which results in less diarrhea $(24,27)$.

It is worth noting that although nausea and vomiting were clustered together as 2 symptoms in the same symptom cluster, the factor load of vomiting in the gastrointestinal symptom group was only 0.68 , while the factor load of nausea was 0.93 . This was consistent with the finding of Smit et al. (28), who observed that the incidence of nausea 


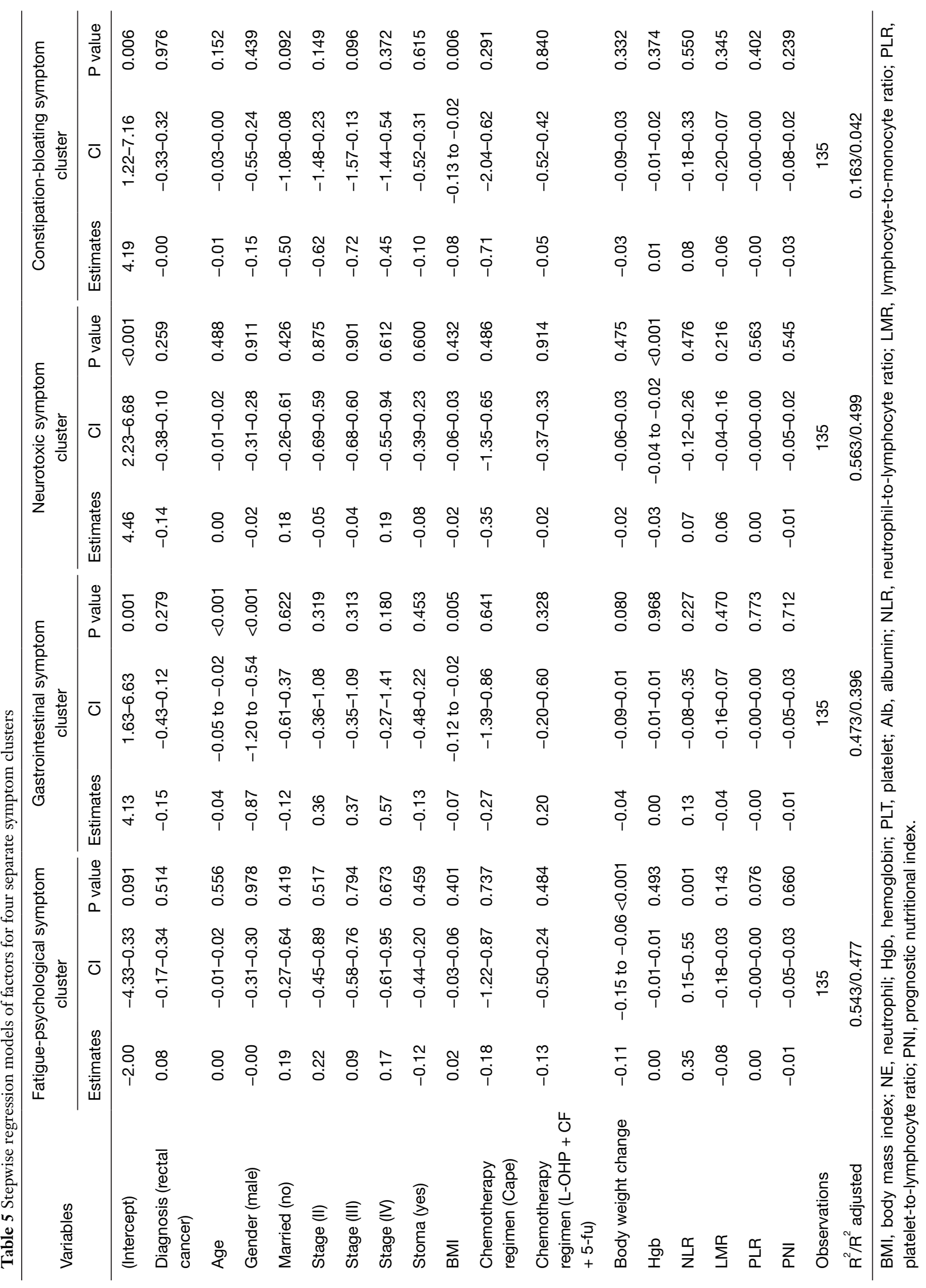




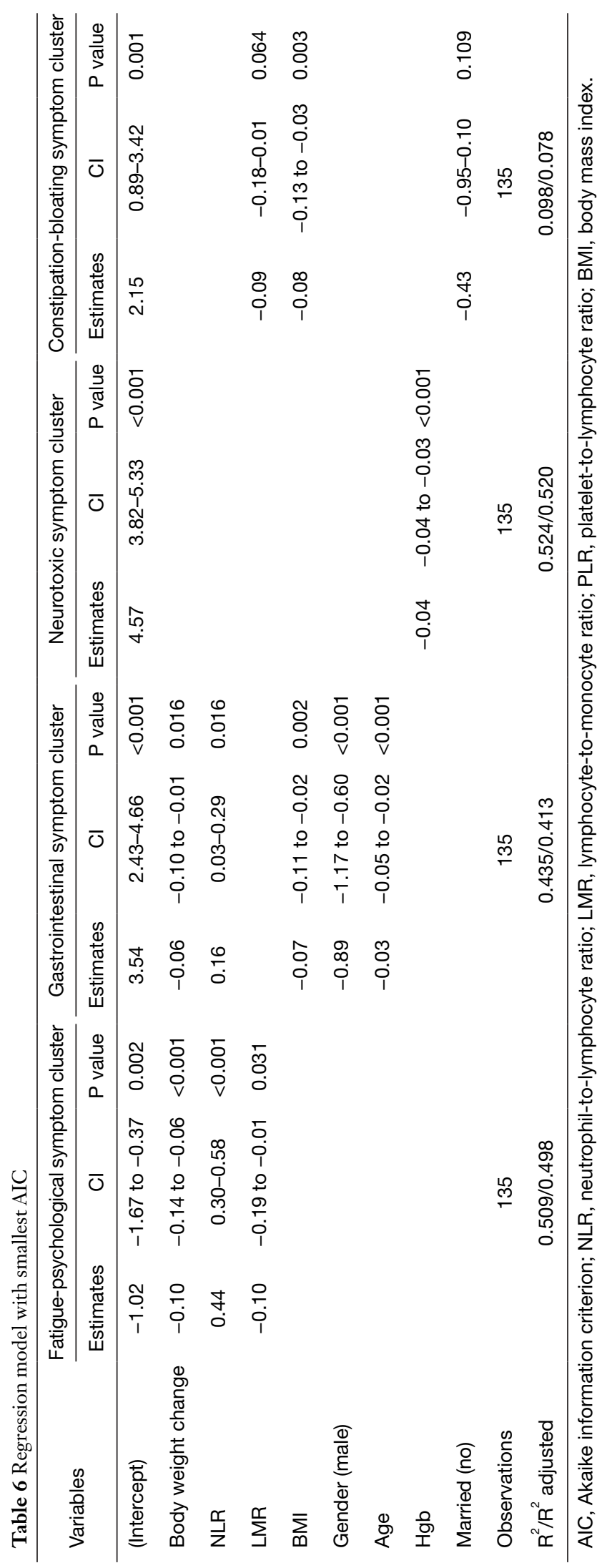

is much higher than that of vomiting. However, in routine studies, vomiting is the initial focus of antiemetic research, and nausea is considered as the secondary end point, so the intervention of nausea is often ignored in clinical work. The symptom cluster provides a new target for clinical nursing. It is a reminder that early intervention for the symptoms that occur in the symptom cluster can help reduce the negative impact of multiple coexisting symptoms.

In a previous study (29), thin frame, female gender, and young age were found to be associated with proneness to nausea and vomiting, and this was accordance with our study. Weight loss characterized by skeletal muscle loss is a hallmark of cancer-related malnutrition, and low muscle mass is associated with increased chemotherapeutic toxicity and impaired physical function (30). In this study, instead of measuring the mass of skeletal muscle, we measured the weight loss after operation, and this suggested that postoperative nutritional support is particularly important for CRC patients.

Most CRC patients benefit from oxaliplatin based chemotherapy, and studies have shown that $94 \%$ of CRC patients experience acute neurotoxicity during adjuvant chemotherapy (31). Due to the short follow up time, the incidence of acute neurotoxicity cluster was low. A related study showed that chemotherapy-induced peripheral neuropathy was associated with emotional symptoms (32), while we did not observe a similar relevance. It is worth mentioning that this study detected a negative correlation between hemoglobin level and neurotoxic cluster, similar to the study of Nilgun Yildirim (33). In combination, the nutritional loss caused by cancer treatment is likely to be the inducing factor of neurotoxic symptoms. However, in this study, sensitivity analysis and internal validation of the model were not performed due to the outcome variable being linear. In addition, as this study was conducted with a limited sample size, multicenter studies with larger sample studies are required to verify these results.

In conclusion, compared with a single symptom, the symptom clusters will significantly reduce the functional status and quality of life of CRC survivors, thus deteriorating patient outcomes (3). By identifying postoperative chemotherapy-related symptom clusters in CRC patients (or symptom cluster indicators), clinicians can better understand CRC patients' experience, and ultimately provide better clinical nursing, thus improving patient compliance with cancer treatment. In this study, we established a convenient and easily available approach to predict chemotherapy related symptom clusters, and the 
Table 7 Correlation analysis of fatigue-psychological symptom cluster, neurotoxic symptom cluster, and nutritional and inflammatory indicators

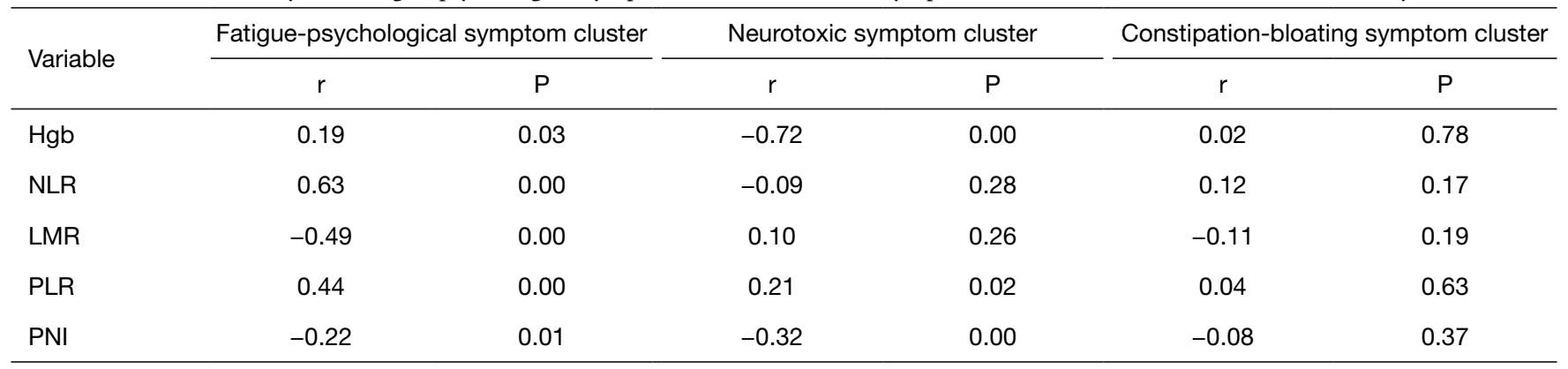

$\mathrm{Hgb}$, hemoglobin; NLR, neutrophil-to-lymphocyte ratio; LMR, lymphocyte-to-monocyte ratio; PLR, platelet-to-lymphocyte ratio; PNI, prognostic nutritional index.

potential indicators will facilitate better understanding of the CRC patients' symptom experience, and finally provide precision clinical nursing and help improve the quality of life of patients. Further longitudinal, multicenter studies and animal experimental studies will help to further explore the mechanism of symptom clusters.

\section{Acknowledgments}

We would like to thank all participants for their cooperation in this study.

Funding: This work was supported by National Natural Science Foundation of China (82172583), Top Talent Support Program for young and middle-aged people of Wuxi Health Committee (BJ2020041), and Key Speciality of Precision Medicine from Wuxi Health and Family Planning Commission (J201807).

\section{Footnote}

Reporting Checklist: The authors have completed the REMARK reporting checklist. Available at https://jgo. amegroups.com/article/view/10.21037/jgo-21-904/rc

Data Sharing Statement: Available at https://jgo.amegroups. com/article/view/10.21037/jgo-21-904/dss

Conflicts of Interest: All authors have completed the ICMJE uniform disclosure form (available at https://jgo.amegroups. com/article/view/10.21037/jgo-21-904/coif). The authors have no conflicts of interest to declare.

Ethical Statement: The authors are accountable for all aspects of the work in ensuring that questions related to the accuracy or integrity of any part of the work are appropriately investigated and resolved. The study was conducted in accordance with the Declaration of Helsinki (as revised in 2013). The study was approved by ethics board of the Affiliated Suqian Hospital of Xuzhou Medical University (No. 2019012). Informed consent was taken from all the patients.

Open Access Statement: This is an Open Access article distributed in accordance with the Creative Commons Attribution-NonCommercial-NoDerivs 4.0 International License (CC BY-NC-ND 4.0), which permits the noncommercial replication and distribution of the article with the strict proviso that no changes or edits are made and the original work is properly cited (including links to both the formal publication through the relevant DOI and the license). See: https://creativecommons.org/licenses/by-nc-nd/4.0/.

\section{References}

1. Arnold M, Sierra MS, Laversanne M, et al. Global patterns and trends in colorectal cancer incidence and mortality. Gut 2017;66:683-91.

2. Han CJ, Yang GS, Syrjala K. Symptom Experiences in Colorectal Cancer Survivors After Cancer Treatments: A Systematic Review and Meta-analysis. Cancer Nurs 2020;43:E132-58.

3. Röhrl K, Guren MG, Astrup GL, et al. High symptom burden is associated with impaired quality of life in colorectal cancer patients during chemotherapy:A prospective longitudinal study. Eur J Oncol Nurs 2020;44:101679.

4. Dodd MJ, Miaskowski C, Paul SM. Symptom clusters and their effect on the functional status of patients with cancer. 
Oncol Nurs Forum 2001;28:465-70.

5. Kim HJ, McGuire DB, Tulman L, et al. Symptom clusters: concept analysis and clinical implications for cancer nursing. Cancer Nurs 2005;28:270-82; quiz 283-4.

6. Lynch Kelly D, Dickinson K, Hsiao CP, et al. Biological Basis for the Clustering of Symptoms. Semin Oncol Nurs 2016;32:351-60.

7. Ji YB, Bo CL, Xue XJ, et al. Association of Inflammatory Cytokines With the Symptom Cluster of Pain, Fatigue, Depression, and Sleep Disturbance in Chinese Patients With Cancer. J Pain Symptom Manage 2017;54:843-52.

8. Li Y, Jia H, Yu W, et al. Nomograms for predicting prognostic value of inflammatory biomarkers in colorectal cancer patients after radical resection. Int J Cancer 2016;139:220-31.

9. Takamizawa Y, Shida D, Boku N, et al. Nutritional and inflammatory measures predict survival of patients with stage IV colorectal cancer. BMC Cancer 2020;20:1092.

10. Park JW, Chang HJ, Yeo HY, et al. The relationships between systemic cytokine profiles and inflammatory markers in colorectal cancer and the prognostic significance of these parameters. Br J Cancer 2020;123:610-8.

11. Yamamoto T, Kawada K, Obama K. Inflammation-Related Biomarkers for the Prediction of Prognosis in Colorectal Cancer Patients. Int J Mol Sci 2021;22:8002.

12. Choi WJ, Cleghorn MC, Jiang H, et al. Preoperative Neutrophil-to-Lymphocyte Ratio is a Better Prognostic Serum Biomarker than Platelet-to-Lymphocyte Ratio in Patients Undergoing Resection for Nonmetastatic Colorectal Cancer. Ann Surg Oncol 2015;22 Suppl 3:S603-13.

13. Fan G, Filipczak L, Chow E. Symptom clusters in cancer patients: a review of the literature. Curr Oncol 2007;14:173-9.

14. Wang XS, Williams LA, Eng C, et al. Validation and application of a module of the M. D. Anderson Symptom Inventory for measuring multiple symptoms in patients with gastrointestinal cancer (the MDASI-GI). Cancer 2010;116:2053-63.

15. Chen RW, Yang SL, Xu ZY, et al. Validation and Application of the Chinese Version of the M. D. Anderson Symptom Inventory Gastrointestinal Cancer Module (MDASI-GI-C). J Pain Symptom Manage 2019;57:820-7.

16. Han CJ, Reding K, Cooper BA, et al. Symptom Clusters in Patients With Gastrointestinal Cancers Using Different Dimensions of the Symptom Experience. J Pain Symptom Manage 2019;58:224-34.

17. Han CJ, Reding K, Cooper BA, et al. Stability of Symptom
Clusters in Patients With Gastrointestinal Cancers

Receiving Chemotherapy. J Pain Symptom Manage 2019;58:989-1001.e10.

18. Thornton LM, Andersen BL, Blakely WP. The pain, depression, and fatigue symptom cluster in advanced breast cancer: covariation with the hypothalamic-pituitaryadrenal axis and the sympathetic nervous system. Health Psychol 2010;29:333-7.

19. Chan GHJ, Chee CE. Making sense of adjuvant chemotherapy in colorectal cancer. J Gastrointest Oncol 2019;10:1183-92.

20. Akagi T, Inomata M. Essential advances in surgical and adjuvant therapies for colorectal cancer 2018-2019. Ann Gastroenterol Surg 2020;4:39-46.

21. Boussios S, Ozturk MA, Moschetta M, et al. The Developing Story of Predictive Biomarkers in Colorectal Cancer. J Pers Med 2019;9:12.

22. Miyamoto Y, Hiyoshi Y, Sawayama H, et al. Precision medicine for adjuvant chemotherapy of resected colorectal cancer. Ann Gastroenterol Surg 2020;4:635-45.

23. Sheu T, Fuller CD, Mendoza TR, et al. Nomogram for predicting symptom severity during radiation therapy for head and neck cancer. Otolaryngol Head Neck Surg 2014;151:619-26.

24. Agasi-Idenburg SC, Thong MS, Punt CJ, et al. Comparison of symptom clusters associated with fatigue in older and younger survivors of colorectal cancer. Support Care Cancer 2017;25:625-32.

25. Steel JL, Kim KH, Dew MA, et al. Cancer-related symptom clusters, eosinophils, and survival in hepatobiliary cancer: an exploratory study. J Pain Symptom Manage 2010;39:859-71.

26. Bower JE. Cancer-related fatigue--mechanisms, risk factors, and treatments. Nat Rev Clin Oncol 2014;11:597-609.

27. Battersby NJ, Bouliotis G, Emmertsen KJ, et al. Development and external validation of a nomogram and online tool to predict bowel dysfunction following restorative rectal cancer resection: the POLARS score. Gut 2018;67:688-96.

28. Smit T, Kotze I, du Plessis J. The incidence of nausea in the absence of vomiting in patients receiving intravenous chemotherapy. Ann Palliat Med 2021;10:2679-86.

29. Takei S, Ishibe A, Watanabe J, et al. Risk factors of chemotherapy-induced nausea and vomiting in patients with metastatic colorectal cancer: a prospective cohort study (YCOG1301). Int J Colorectal Dis 2020;35:2323-9.

30. Ryan AM, Prado CM, Sullivan ES, et al. Effects of weight loss and sarcopenia on response to chemotherapy, quality 
of life, and survival. Nutrition 2019;67-68:110539.

31. Soveri LM, Lamminmäki A, Hänninen UA, et al. Longterm neuropathy and quality of life in colorectal cancer patients treated with oxaliplatin containing adjuvant chemotherapy. Acta Oncol 2019;58:398-406.

32. Lu LC, Tsay SL, Chang SY, et al. Daily activity, mood, and quality of life in colorectal cancer patients with

Cite this article as: Li N, Lu J, Xia D, Jiang X, Wen X, Qin X, Chen Y, Wang T. Serum biomarkers predict adjuvant chemotherapy-associated symptom clusters in radical resected colorectal cancer patients. J Gastrointest Oncol 2022;13(1):197209. doi: 10.21037/jgo-21-904 chemotherapy-induced peripheral neuropathy: A mediation effect analysis. Cancer Med 2019;8:963-71.

33. Yildirim N, Cengiz M. Predictive clinical factors of chronic peripheral neuropathy induced by oxaliplatin. Support Care Cancer 2020;28:4781-8.

(English Language Editor: J. Jones) 